\title{
INVESTIGATING THE ACCURACY OF GRAVITY FIELD MODELS USING SATELLITE LASER RANGING DATA
}

\author{
M.C. BOTAI AND L. COMBRINCK \\ Space Geodesy Programme, HartRAO, P.O. Box 443, Krugersdorp, 1740, South Africa \\ Department of Geography, Geoinformatics and Meteorology, University of Pretoria, South Africa \\ e-mail: christina@hartrao.ac.za; ludwig@hartrao.ac.za
}

(C) 2011 December Geological Society of South Africa

\begin{abstract}
Continuous tracking of geodetic satellites using the Satellite Laser Ranging technique has provided unprecedented opportunity in long- to medium-wavelength gravity field modelling. Numerous gravity field models have been derived from such observations and have been made freely available to the science community for research purpose. The accuracy of most of the latest gravity field models in terms of precise orbit determination is currently at $\mathrm{cm}$ level. Improvement in the Earth gravity field modelling is anticipated as quantitative and qualitative data (in particular from low earth orbit satellites) become available in the future. Such expectations require that the accuracy and precision of existing gravity field models be assessed. The validation of gravity field models in terms of satellite orbit determination is often based on the difference between the observed and computed range. The resulting range residuals are considered an important index when determining the accuracy of the gravity models and hence the satellite orbits. In this contribution we investigate the general improvement in gravity field modelling over a period of 15 years. The orbit accuracy of twelve gravity field models (both satellite-only and combined models) were assessed by analysing seven months of data from LAGEOS 1 and 2 using HartRAO analysis software. Results show that the gravity field models developed over the years have improved by at least a factor of 2 since 1990, considering improvement in O-C residuals.
\end{abstract}

\section{Introduction}

Global Geo-potential Models (GGMs) allow researchers to probe the long- to medium-wavelength components (half-wavelengths longer than $200 \mathrm{~km}$, or spherical harmonic degrees 2 to 100) of the Earth's gravitational field. Such contributions are often recovered from satellite tracking data through SLR observations (Biancale, et al., 2000 and Reigber et al., 2006). The Satellite Laser Ranging (SLR) technique allows measurement of the distance between artificial satellites orbiting the earth and ground stations. Ultra-short pulses of light are fired from a network of ground stations to target satellites equipped with retro-reflectors and reflected back to the ground station where the Time-Of-Flight (TOF) is determined by the SLR time measurement systems. The TOF allows the distance between the SLR station and the retroreflector satellite to be measured as

$$
\mathrm{d}=\frac{\mathrm{ct}}{2}
$$

where $\mathrm{c}$ is the speed of light and $\mathrm{t}$ is the TOF.

The motion of an orbiting satellite is perturbed by various forces such as gravitational, non-gravitational and empirical or un-modelled forces. If a satellite is assumed to be in an inertial reference frame its motion can be expressed as

$$
\ddot{\bar{r}}=\bar{a}_{g}+\bar{a}_{n g}+\bar{a}_{e m p}
$$

where $\bar{r}$ is the position vector of the centre of mass of the satellite, $\bar{a}_{g}$ is the sum of the gravitational forces acting on the satellite, $\bar{a}_{n g}$ is the sum of the nongravitational forces acting on the surface of the satellite and $\bar{a}_{\text {emp }}$ is the unmodelled forces which act on the satellite due to either a functionally incorrect or incomplete description of the various forces acting on the satellite. The gravitational forces, $\bar{a}_{g}$ acting on the satellite are composed of a series of perturbations expressed as

$$
\overline{\mathrm{a}}_{g}=\overline{\mathrm{P}}_{\text {geo }}+\overline{\mathrm{P}}_{\mathrm{set}}+\overline{\mathrm{P}}_{\mathrm{ot}}+\overline{\mathrm{P}}_{\mathrm{rd}}+\overline{\mathrm{P}}_{\mathrm{smp}}+\overline{\mathrm{P}}_{\mathrm{rel}}
$$

where, $\overline{\mathrm{P}}_{\text {geo }}$ is the geopotential force due to the gravitational attraction of the Earth, $\overline{\mathrm{P}}_{\text {set }}$ and $\overline{\mathrm{P}}_{\mathrm{ot}}$ defines perturbations due to solid Earth tides and ocean tides respectively, $\overline{\mathrm{P}}_{\mathrm{rd}}$ describes perturbations due to the rotational deformation, $\overline{\mathrm{P}}_{\mathrm{smp}}$ are perturbations due to the Sun, Moon and planets and $\overline{\mathrm{P}}_{\text {rel }}$ describes perturbations due to general relativity. The nongravitational forces acting on an orbiting satellite are given by

$$
\overline{\mathrm{a}}_{\text {ng }}=\overline{\mathrm{P}}_{\text {drag }}+\overline{\mathrm{P}}_{\text {solar }}+\overline{\mathrm{P}}_{\text {earth }}+\overline{\mathrm{P}}_{\text {thermal }}
$$

where $\overline{\mathrm{P}}_{\text {drag }}$ is the atmospheric drag acting on a satellite, $\overline{\mathrm{P}}_{\text {solar }}$ is due to solar radiation pressure, $\overline{\mathrm{P}}_{\text {earth }}$ describes perturbation due to solar radiation pressure reflected from the earth to an orbiting satellite, $\bar{P}_{\text {thermal }}$ is the perturbation due to thermal radiation imbalance resulting from non-uniform temperature on different satellite surfaces.

The geopotential function, $\mathrm{V}$, of the solid-mass distribution of the Earth in a body-fixed reference frame 
is often reconstructed by using expanded spherical harmonics (i.e. the eigenfunctions of the spherical form of Laplace's equations), given for example by

$\mathrm{V}(\mathrm{r}, \phi \lambda)=\frac{\mathrm{GM}_{\mathrm{e}}}{\mathrm{r}}+\frac{\mathrm{GM}_{\mathrm{e}}}{\mathrm{r}} \sum_{\mathrm{n}=1}^{\infty} \sum_{\mathrm{m}=0}^{1}\left(\frac{\mathrm{a}_{\mathrm{e}}}{\mathrm{r}}\right)^{1} \overline{\mathrm{P}}_{\mathrm{nm}}(\sin \varphi)\left[\mathrm{C}_{\mathrm{nm}} \cos \mathrm{m} \lambda+\overline{\mathrm{S}}_{\mathrm{nm}} \sin \mathrm{m} \lambda\right]$

Here, $\mathrm{GM}_{\mathrm{e}}$ is the gravitational constant of the Earth, $\mathrm{a}_{\mathrm{e}}$ is the mean equatorial radius of the Earth $(6378.1 \mathrm{~km})$, $(r, \phi, \lambda)$ are the radial distance from the centre of mass of Earth, geocentric latitude and the longitude of the satellite respectively, $\overline{\mathrm{P}}_{\mathrm{nm}}(\sin \varphi)$ is the fully-normalised associated Legendre function of degree $n$ and order $m$, $\left\{\overline{\mathrm{C}}_{\mathrm{nnmm}} \overline{\mathrm{S}}_{\mathrm{nm}}\right\}$ are normalised spherical harmonic coefficients (Stock's coefficients) of degree $n$ and order m. A typical geopotential model is often described by these spherical harmonic coefficients. The values of $\left\{\overline{\mathrm{C}}_{n n m m} \overline{\mathrm{S}}_{\mathrm{nm}}\right\}$ coefficients decrease as the degree increase. For satellite-based global gravity field models the accuracy of the lower degree coefficients is typically higher than the higher degree/order coefficients.

A number of spherical harmonic models have been developed over the years. The development of such models could be attributed to the availability of data as well as the SLR tracking data of multiple satellites. For instance, some of the gravity models were developed by including surface gravity measurements, satellite-to-satellite tracking and satellite radar altimeter measurements of the ocean surface. The accuracy of the gravity field models depend on numerous factors such as data availability, quality, type and geographical coverage. Difficulties in modelling the non-gravitational forces for most of the geodetic satellites (in particular the low earth orbits) also limit the plausibility of achieving significant improvement in the gravitational field modelling (Biancale et al., 2000). Recent low orbit satellite missions such CHAllenging Minisatellite Payload as (CHAMP), Gravity Recovery and Climate Experiment (GRACE) and Gravity field and steady-state Ocean Circulation Explorer (GOCE) are designed to resolve the long-wavelength part of the gravity field and hence provide unprecedented accuracy. In contrast to the sporadic tracking by SLR the three satellite missions carry GPS receivers on board that allow continuous orbit tracking. Furthermore, these satellites are equipped with accelerometers which provide direct measurements of the non-conservative forces (e.g. air-drag). In the case of GOCE, six accelerometers are installed in a gradiometer arrangement which additionally allows for direct measurement of the Earth's gravity gradients which gives an improvement in the medium wavelength part of the gravity.

The latest gravity field models which are derived from individual or combination of improved data from CHAMP and GRACE have demonstrated significant improvement (up to cm-level accuracy) in the determination of gravity anomalies, geoidal heights and precise orbit determination (Reigber et al., 2004).
Improvements in gravity field modelling are anticipated as quantitative data become available in the future. In particular, the employment of longer data spans from CHAMP, GRACE and GOCE with improved processing algorithms and empirical models are expected to increase the resolution and further improve the accuracy in gravity field models. Such expectations require that the accuracy and precision of existing gravity field models be assessed and validated. This can be achieved by evaluating their performance in terms of precise satellite orbit determination. Different methods have been used in satellite orbit determination. The most commonly used are the kinematic and dynamic methods. The kinematic method is a geometrical method which does not consider the dynamic property of an orbiting satellite. This method relies on observational data (kinematic positions of an orbiting satellite) and hence assumes the availability of highly accurate measurements and high data sampling rate. The dynamic method uses force and satellite models to compute the satellite's acceleration. Satellite position as a function of time is then computed by using either analytical or numerical integration. Selected parameters of the force models acting on the satellite may be adjusted along with an initial satellite position and velocity in order to minimise the observation residuals in a least-squares sense.

In this report, an assessment of the accuracy of gravity field models using SLR data is presented. The results are based on the dynamic orbit fits of O-C residuals computed using the SLR analysis software developed at Hartebeesthoek Radio Astronomy Observatory (HartRAO), South Africa.

\section{Data and method}

The SLR data used in the present study covered the period from the 1st December 2005 to 30th June 2006. The analysed data sets were retrieved from the NASA Crustal Dynamic Data Information System (CDDIS) which collects data from different tracking stations, archive and distribute it to the science community for research purposes. The data from different stations arrive at CDDIS as huge measurements with varying delays. Since it is impossible to process the whole data, a sampling is performed to compress a certain number of measurements into one dataset over a satellite pass. This data compression process leads to the formation of normal points over a certain pass and is computed using the formulation

$$
\mathrm{NPR}_{\mathrm{i}}=\left\{\frac{\mathrm{C} \times \mathrm{NP}_{\mathrm{TOF}_{\mathrm{i}}}}{10^{12}}\right\}\left\{2-\Delta \mathrm{a}_{\mathrm{i}}+\Delta \mathrm{CoM}_{\mathrm{i}}-\Delta \mathrm{R}_{\mathrm{b}_{\mathrm{i}}}-\Delta \mathrm{GR}_{\mathrm{i}}-\Delta \epsilon_{\mathrm{i}}\right\}^{-1} .
$$

Equation 6 takes into account the full rate measurements $\mathrm{NP}_{\text {TOF, }}$ atmospheric effects $(\Delta a)$, Centre-of-Mass $(\triangle C O M)$, correction of the satellite, station range bias $\left(\Delta R_{b}\right)$ and a relativistic correction $(\Delta G R)$. In addition, 
Table 1. Constants, reference frames and empirical models used in the SLR data processing

\begin{tabular}{|c|c|}
\hline Inertial reference frame & $\mathrm{J} 2000$ \\
\hline Terrestrial reference frame & ITRF2000 epoch 1997.0 \\
\hline Pole-tide correction (station position) & IERS 2003 \\
\hline Relativity (space-time curvature) & IERS 2003 \\
\hline Earth-tide correction (station position) & Petrov (2005) \\
\hline Earth-tide acceleration of satellite & Rizos and Stolz (1985) \\
\hline Ocean loading correction (station position) & Scherneck (1991) \\
\hline Atmospheric loading & Special Buro for Loading, IERS \\
\hline Reference epoch & 1997 \\
\hline Tectonic plate model & ITRF2000 velocity field \\
\hline Earth orientation & $\begin{array}{l}\text { a-priori Earth orientation parameters and UTC-UT1 values as per IERS Bulletin B } \\
\text { extrapolated to observation epoch }\end{array}$ \\
\hline O-C outlier rejection & selectable \\
\hline Data rejection & 10 degrees elevation \\
\hline Range bias & Enabled \\
\hline Time bias & Enabled \\
\hline Satellite centre-of-mass offset & $251 \mathrm{~mm}$, ILRS standard value (Otsubo and Appleby, 2003) \\
\hline
\end{tabular}

$N P R$ and $\Delta \varepsilon$ are the normal point range and the correction for unknown systematic and random errors respectively. The normal point (NP) data are produced from the normal point range by using the formulation

$$
\mathrm{NP}=\mathrm{O}-\mathrm{FR}-\overline{\mathrm{FR}}
$$

where $\mathrm{O}$ represents the observations, $\overline{\mathrm{FR}}$ is the fit residuals of the observations (subdivided into bins) and is the mean value of the fit residuals for a specific bin.

The seven months of normal point data from LAGEOS 1 and 2 collected from an average of 22 SLR stations of the International Laser Ranging Service (ILRS) network (Pearlman et al., 2000) were analysed using the HartRAO analysis software described in Combrinck and Suberlak (2007). During the analysis the orbital arc length was fixed to 24 hours for all the observations. Station displacement models for solid earth tides (Petrov, 2005), ocean tides (Scherneck, 1991), atmospheric tides and pole tides (IERS 2003) were included in the HartRAO software system. In addition, perturbations from the gravitational attractions of the Moon, the Sun and the eight major planets were taken into account following the Jet Propulsion Laboratory (JPL) DE-405 planetary ephemeris (Standish, 1998). Surface forces such as atmospheric drag, solar and earth radiation pressure were also implemented in the software. All these models aimed to achieve better solutions thereby minimising the $\mathrm{O}-\mathrm{C}$ residuals. The parameters considered during data processing are listed in Table 1. In the analysis, the effects on the transformation of the Center-of-Mass (CoM) corrections of satellites were not considered although it is a selectable option in the software.

The HartRAO analysis program computes several output parameters. These include O-C residuals, time and range biases, laser station coordinates and their velocities. In addition, the program estimates solve-for parameters such as $\mathrm{J}_{2}-\mathrm{J}_{5}, \mathrm{~S}_{21}$ and $\mathrm{C}_{21}$. In this paper we focus on the computed $\mathrm{O}-\mathrm{C}$ residuals to assess the accuracy of GGMs as applied to precise orbit determination. The analysis only considers spherical harmonic coefficients of degree and order 20. These lower order coefficients are applicable to satellites at the orbital height of LAGEOS.

\section{Geo-potential models evaluated}

A total of twelve gravity field models were considered in this study. The present analysis selected gravity field models from both the satellite-only and the combined (satellite and terrestrial data) classes. A brief description of each model follows and a summary is given in Table 2 .

\section{AIUB-GRACE01S}

AIUB-GRACE01S was a GRACE-only static gravity field model complete to degree and order 120 in terms of spherical harmonics. The model was generated from GPS satellite-to-satellite tracking data and K-band range rate measurements out of the period from January 2003 to December 2003 using the Celestial Mechanics approach (Jaeggi et al., 2008).

\section{EIGEN-5C and EIGEN-5S}

The European Improved Gravity model of the Earth by New techniques (EIGEN)-5C was derived complete to degree and order 360. The model was computed in a joint collaboration between GFZ Potsdam and CNES/GRGS Toulouse. The EIGEN-5C model incorporated satellite tracking data from GRACE and LAGEOS, gravimetry and altimetry surface data. The data were combined using accumulation of normal equations obtained from observation equations for the spherical harmonic coefficients (Foerste et al., 2008). The satelliteonly model, EIGEN-5S, was computed complete to degree and order 150. The model incorporated GRACE 
Table 2. Global Geopotential Models evaluated in this study.

\begin{tabular}{|c|c|c|c|c|}
\hline Model & Year & Degree & Data & Reference \\
\hline AIUB-GRACE01S & 2008 & 120 & $\mathrm{~S}$ & Jaeggi et al. (2008) \\
\hline EIGEN-5C & 2008 & 360 & $\mathrm{~S}, \mathrm{G}, \mathrm{A}$ & Foerste et al. (2008) \\
\hline EIGEN-5S & 2008 & 150 & $\mathrm{~S}$ & Foerste et al. (2008) \\
\hline GGM03C & 2007 & 360 & $\mathrm{~S}, \mathrm{G}, \mathrm{A}$ & Tapley et al. (2007) \\
\hline EIGEN-GL04S1 & 2006 & 150 & $\mathrm{~S}$ & Foerste et al. (2006) \\
\hline EIGEN-CG03C & 2005 & 360 & $\mathrm{~S}, \mathrm{G}, \mathrm{A}$ & Foerste et al. (2005) \\
\hline EIGEN1 & 2002 & 119 & S & Reigber et al. (2003) \\
\hline GRIM5C1 & 1999 & 120 & $\mathrm{~S}, \mathrm{G}, \mathrm{A}$ & Gruber et al. (2000) \\
\hline EGM96 & 1996 & 360 & EGM96S,G,A & Lemoine et al. (1998) \\
\hline JGM3 & 1994 & 70 & $\mathrm{~S}, \mathrm{G}, \mathrm{A}$ & Tapley et al. (1996) \\
\hline OSU91A & 1991 & 360 & GEMT2,A,G & Rapp et al. (1991) \\
\hline GRIM4C1 & 1990 & 50 & $\mathrm{~S}, \mathrm{G}, \mathrm{A}$ & Schwintzer et al. (1993) \\
\hline
\end{tabular}

Data: $\mathrm{S}=$ Satellite tracking data $\mathrm{G}=$ Terrestrial gravity data $\mathrm{A}=$ Altimetry data.

data used in the computation of EIGEN-5C model and some extended data from GRACE and LAGEOS covering the period between mid 2002 and end of 2007 (Foerste et al., 2008).

\section{GGM03C}

The combined GGM03C model reported by Tapley et al., 2007 was computed from the combination of satellite-only gravity field model GGM03S with land and ocean gravity data. The model was derived complete to degree and order of 360 .

\section{EIGEN-GL04S1}

The satellite-only model EIGEN-GL04S1 (Foerste et al., 2006) was computed complete to degree and order 150 in terms of spherical harmonics. The model incorporated solutions from EIGEN-GRACE04S and EIGEN-GL04S gravity field models.

\section{EIGEN-CG03C}

The GRACE based gravity field model, EIGEN-CG03C, was generated by the GFZ-GRGS cooperation. The model was an upgrade of the combined gravity field model, EIGEN-CG01C reported by Foerste et al., (2008). The EIGEN-CG03C model was computed by combining CHAMP data with gravimetry and altimetry data using normal equations obtained from observation equations for the spherical harmonic coefficients (Foerste et al., 2008).

\section{EIGEN1}

The EIGEN1 model reported by Reigber et al., (2003) was derived in a joint German-French collaboration using three months of data collected from the CHAMP mission. The model was computed up to maximum degree and order 119 in terms of spherical harmonics.

\section{GRIM5C1}

The GRIM5C1 gravity field model reported by Gruber et al., (2000) was derived in a German-French joint collaboration between GFZ Potsdam and GRGS
Toulouse. The model was computed up to degree and order 120. It incorporated terrestrial and airborne mean gravity anomalies, altimetric gravity anomalies from NIMA and mean gravity anomalies derived from the GRIM5S1 model.

\section{EGM96}

EGM96 was a spherical harmonic model of the earth's gravitational potential to degree and order of 360 . The model was derived through collaboration between NASA Goddard Space Flight Center (GSFC), National Imagery and Mapping Agency (NIMA), and the Ohio State University (OSU). The EGM96 model was developed by combining surface gravity data, ERS-1 and GEOSAT altimeter-derived anomalies, satellite tracking data from SLR, GPS, NASA's tracking and data relay satellite system (TDRSS), the French DORIS system, the US Navy TRANET Doppler tracking system and direct altimeter ranges from T/P, ERS-1, and GEOSAT (Lemoine et al., 1998). The coefficients were a blend of three computational procedures. From degree 2 to 70 the coefficients were based on a least squares adjustment involving satellite tracking data, terrestrial data, direct altimeter data, and fill-in anomalies in areas lacking data. The coefficients from degree 71 to 359 were taken from a block diagonal combination solution (Pavlis et al., 1996) using normal equations derived from the satellite tracking data as a priori values. The coefficients of degree 360 were taken from a quadrature combination solution using the a priori satellite model and a global anomaly set.

\section{JGM3}

The JGM3 model was released in 1994 by Tapley et al., (1996). The model was estimated from surface gravity data used in the computation of the JGM1 model. The solution was combined with SLR data from GEOdetic SATellite (GEOSAT) and ERS-1 satellites, DORIS tracking data from TOPEX/Poseidon (T/P) and SPOT2 and GPS data. The JGM3 model was derived complete to degree and order 70 . 


\section{OSU91A}

The gravity field model OSU91A computed by Rapp et al. (1991) to order and degree 360 involved two computational procedures. The first was a computation of the coefficients up to degree and order 50 achieved by combining the solution from the GEM-T2 model with surface data and GEOSAT altimeter data. The coefficients from degree 51 to 360 were derived from a combined solution of gravity anomalies obtained from terrestrial data, altimeter derived anomalies, and the topographic/isostatic anomalies.

\section{GRIM4C1}

The GRIM4C1 combined gravity field model was reported by Schwintzer et al. (1993). This model was computed in a joint collaboration between DGFI and GRGS. The GRIM4C1 model was derived up to degree and order 50 in terms of spherical harmonics. It incorporated GRIM4S1 satellite-solution, mean gravity anomalies and Seasat altimeter derived mean geoid undulations.

\section{Results and discussion}

Figure 1 contains the mean O-C RMS of the evaluated gravity field models plotted against their year of computation. It can be seen that there has been an improvement in gravity field modelling between 1990 and 2008. This is confirmed by a parabolic line obtained when fitting the O-C residual errors from LAGEOS 1 . The orbit fitting for LAGEOS 2 also illustrates a parabolic line which flattens to a slight curve for models derived in 2002 and 2007. Variations between models in the latter period may be influenced by outliers related to weak station geometry and lack of data during some days. Relevant statistical analyses of the models tested are contained in Table 3. In Table 3, the oldest model, GRIM4C1, which was released in 1990, exhibits the highest RMS errors of $9.3 \mathrm{~cm}$ and $6.6 \mathrm{~cm}$ for LAGEOS 1 and 2 respectively. The models released from 1996 (EGM96) seem to remain at approximately the same level, although there are many specific differences (e.g. type of data used, degree and order of coefficients) amongst these later models. Since the addition of CHAMP and GRACE data is expected to yield an improvement in gravity field models modelling errors might be dominating in our analysis. In the case where modelling errors dominate, the inaccuracies caused by the modelling of other perturbing forces are greater than the contribution from gravity field models and thus obscure the improvement in the gravity modelling. In general, the evaluated gravity field models show an improvement by a factor of 2 since 1990. The GRACEonly gravity field model, AIUB-GRACE01S yields the lowest $\mathrm{O}-\mathrm{C}$ results therefore it seems to be the most accurate in terms of our evaluation.

\section{Conclusion}

Twelve gravity field models released between 1990 and 2008 were analysed to investigate general improvement in gravity field determination. Accuracy of the selected gravity field models were assessed in terms of precise orbit determination by comparing their O-C residuals. The results indicate that there has been an improvement in the development of gravity field models over the period of evaluation. The evaluated models show an improvement by a factor of at least 2 since 1990 in terms of O-C residuals. Since 1996 (EGM96), models seemed to be accurate at approximately the same level, at least to the sensitivity of our $\mathrm{O}-\mathrm{C}$ tests, although there are many specific differences amongst these later models.

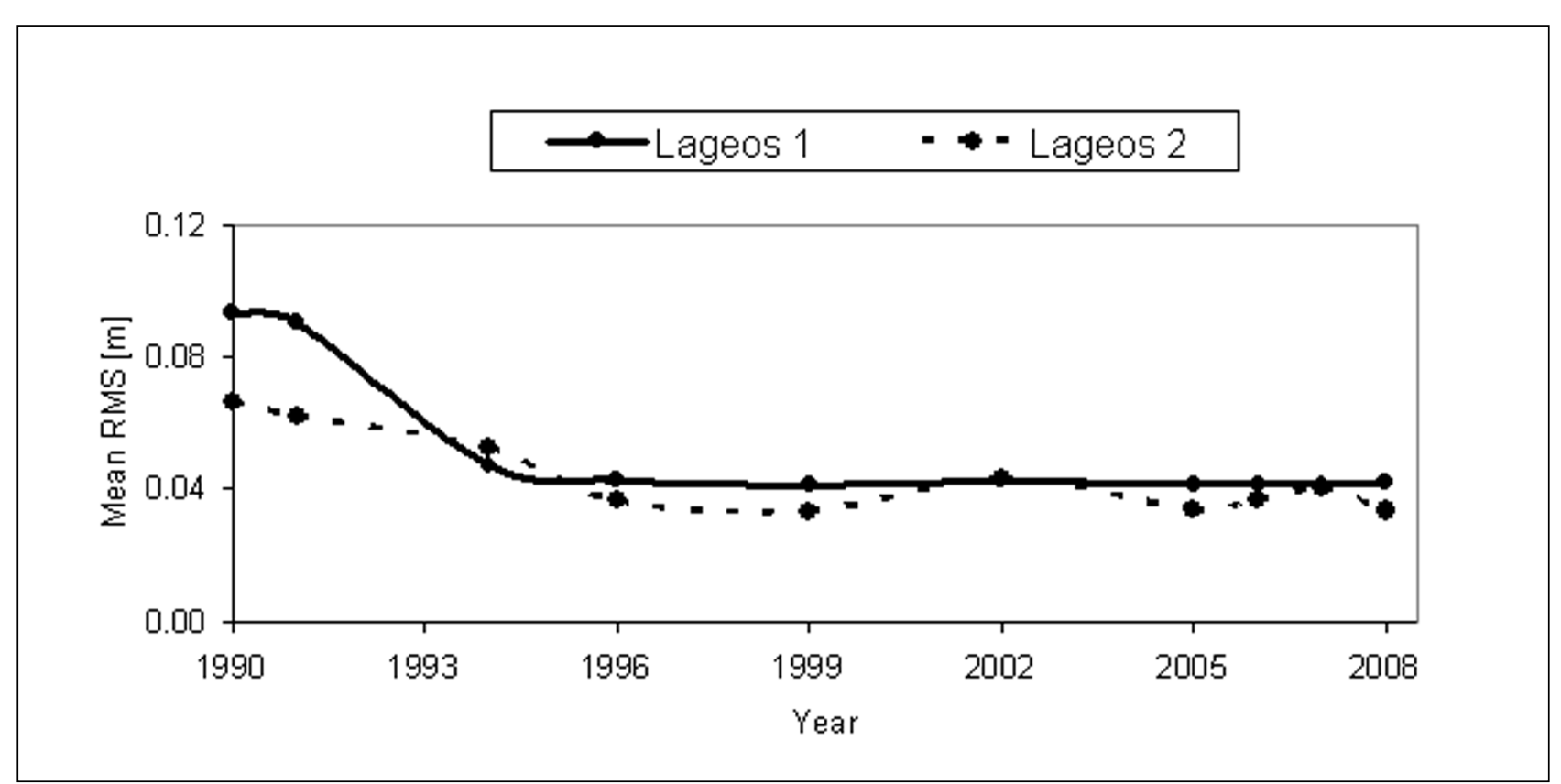

Figure 1. Time series of the mean RMS values for the evaluated Global Geopotential Models. 
Table 3. Comparative accuracies of the evaluated gravity field models in terms of $\mathrm{O}-\mathrm{C}$ residuals

\begin{tabular}{lccc}
\hline Model & $\begin{array}{c}\text { Year of } \\
\text { publication }\end{array}$ & $\begin{array}{c}\text { Mean RMS } \\
\text { [cm] } \\
\text { LAGEOS 1 }\end{array}$ & $\begin{array}{c}\text { Mean RMS } \\
\text { [cm] } \\
\text { LAGEOS 2 }\end{array}$ \\
\hline AIUB-GRACE01S & 2008 & 4.17 & 3.33 \\
EIGEN-5C & 2008 & 4.19 & 3.41 \\
EIGEN-5S & 2008 & 4.2 & 3.37 \\
GGM03C & 2007 & 4.17 & 4 \\
EIGEN-GL04S1 & 2006 & 4.16 & 3.65 \\
EIGEN-CG03C & 2005 & 4.14 & 3.38 \\
EIGEN1 & 2002 & 4.25 & 4.33 \\
GRIM5C1 & 1999 & 4.13 & 3.34 \\
EGM96 & 1996 & 4.27 & 3.64 \\
JGM3 & 1994 & 4.74 & 5.26 \\
OSU91A & 1991 & 9.04 & 6.22 \\
GRIM4C1 & 1990 & 9.31 & 6.64 \\
\hline
\end{tabular}

Long term processing is needed to confirm the current results and additional historical and new models including EGM2008 will be added. The model AIUBGRACE01S which yields the lowest $\mathrm{O}-\mathrm{C}$ results seems to be the most accurate in terms of our evaluation.

\section{Acknowledgements}

The data employed in this study were obtained from NASA Crustal Dynamics Data Information System (CDDIS) as provided by SLR stations within the ILRS (Pearlman et al., 2002). The authors wish to thank the reviewers for their constructive and detailed comments that helped to improve the quality of the manuscript. This is Inkaba yeAfrica publication 57.

\section{References}

Biancale, R., Balmino, G., Lemoine, J.-M., Marty, J.-C., Moynot, B., Barlier, F., Exertier, P., Laurain, O., Gegout, P., Schwintzer, P., Reigber, C., Bode, A., König, R., Massmann, F.-H., Raimondo, J.-C., Schmidt, R., Zhu, S.Y., 2000. A new global Earth's gravity field model from satellite orbit perturbations: GRIM5-S1. Geophysical Research Letters, 27, 3611-3614.

Combrinck, L., and Suberlak, V., 2007. Earth-tide as parameter of crustal motion to correction for SLR station displacement: South African Journal of Geology, 110, 203-210.

Foerste, Ch., Flechtner, F., Schmidt, R., Stubenvoll, R., Rothacher, M., Kusche, J., Neumayer, H., Biancale, R., Lemoine, J.M., Barthelmes, F., Bruinsma, S., Koenig, R., Meyer, Ul., 2008. EIGEN-GL05C - A new global combined high-resolution GRACE-based gravity field model of the GFZ-GRGS cooperation, Geophysical Research Abstracts, 10, EGU2008-A-03426.

Foerste C., Flechtner, F., Schmidt, R., Koenig R., Meyer, U., Stubenvoll, R., Rothacher, M., Barthelmes, F., Neumayer, H., Biancale, R., Bruinsma, S., Lemoine, J.M., Loyer, S., 2006. Global mean gravity field models from combination of satellite mission and altimetry/gravimetry surface data, Geophysical Research Abstracts, 8, 03462.

Gruber, Th., Bode, A., Reigber, Ch., Schwintzer, P. (GFZ Potsdam), Biancale, R., Balmino, G., Lemoine, J.-M. (CNES/GRGS, Toulouse, France), 2000. GRIM5-C1: Combination solution of the global gravity field to degree and order 120, Geophysical Research Letters, 27, 4005-4008.

IERS Conventions, 2003. Dennis D. McCarthy and Gérard Petit. (IERS Technical Note; 32) Frankfurt am Main: Verlag des Bundesamts für Kartographie und Geodäsie, 72-84. http://www.iers.org/iers/ publications/tn/tn32
Jaeggi, A., Beutler, G., Mervart, L., 2008. GRACE gravity field determination using the Celestial Mechanics approach - First results, presented at the IAG Symposium on "Gravity, Geoid, and Earth Observations 2008" Chania/Greece, 177-184

Lemoine, F.G., Kenyon, S.C., Factor, J.K., Trimmer, R.G., Pavlis, N.K., Chinn, D.S., Cox, C.M., Klosko, S.M., Luthcke, S.B., Torrence, M.H., Wang, Y.M., Williamson, R.G., Pavlis, E.C., Rapp, R.H., Olson, T.R., 1998. The Development of the Joint NASA GSFC and the National Imagery and Mapping Agency (NIMA) Geopotential Model EGM96; NASA Technical Paper NASA/TP 1998206861, Goddard Space Flight Center, Greenbelt, USA, $1-9$.

Otsubo, T., and Appleby, G., 2003. System-dependent centre-of-mass correction for spherical geodetic satellites, Journal of Geophysical Research, 108, B4, 2201.

Pavlis, N.K., Chan, J.C., and Lerch, F., 1996. Alternative estimation technique for global high-degree gravity modelling. In: R.H. Rapp, A.A. Cazenare, R.S. Nerem, (Editors) Proc IAG Symp 116, Global gravity field and its temporal variations, Springer, Berlin, Germany, 111-120.

Pearlman, M. R., Degnan, J. J., and Bosworth, J. M., 2002. The International Laser Ranging Service, Advances in Space Research, 30, 135-143.

Petrov, L., 2005. Software sotid for computation of site displacements due to the solid Earth tides, available from http://gemini.gsfc.nasa.gov/sotid.

Rapp, R.H., Wang, Y.M., and Pavlis, N.K., 1991. The Ohio State 1991 Geopotential and Sea Surface Topography Harmonic Coefficient Models, Report 410, Department of Geodetic Science and Surveying, The Ohio State University, Columbus, U.S.A. 43210-1247.

Reigber, Ch., Schwintzer, P., Stubenvoll, R. Schmidt, R., Flechtner, F., Meyer, U., Koenig, R., Neumayer, H., Foerste, Ch., Barthelmes, F., Zhu, S.Y., Balmino, G., Biancale, R., Lemoine, J.-M., Meixner, H., Raimondo, J.C., 2006. A High Resolution Global Gravity Field Model Combining CHAMP and GRACE Satellite Mission and Surface Data: EIGEN-CG01C, Scientific Technical Report STR06/07, GeoForschungsZentrum Potsdam.

Reigber Ch., Schmidt R., Flechtner F., Koenig R., Meyer U., Neumayer K.-H., Schwintzer P. and Zhu S.Y., 2004. An Earth gravity field model complete to degree and order 150 from GRACE: EIGEN-GRACE02S, Journal of Geodynamics 39 (2005), 1-10.

Reigber, Ch., Balmino, G., Schwintzer, P., Biancale, R., Bode, A., Lemoine, J.-M., Koenig, R., Loyer, S., Neumayer, H., Marty, J.-C., Barthelmes, F., Perosanz, F., Zhu, S.Y., 2003. Global Gravity Field Recovery Using Solely GPS Tracking and Accelerometer Data From CHAMP. In: G. Beutler, M.R. Drinkwater, R. Rummel and R. von Steiger (Editors). Earth Gravity Field from Space - From Sensors to Earth Sciences. Proceedings of an ISSI Workshop 11-15 March 2002, Bern Switzerland, Space Science Reviews, 108, 55-66.

Rizos, C. and Stolz, A., 1985. Force modelling for GPS satellites orbits. 1st International Symposium on Precise Positioning with GPS, Rockville, Maryland, United States of America, 1, 87-98.

Scherneck, H.G., 1991. A parameterized solid Earth tide model and ocean tide loading effects for global geodetic baseline measurements, Geophysical Journal International, 106, 677-694.

Standish, E.M., 1998. JPL Planetary and Lunar Ephemerides DE405/LE405, Jet Propulsion Laboratory Interoffice Memorandum, IOM 312.F-98-048.

Schwintzer, P., Reigber, C., Bode, A., Chen, Z., Massman, F.H., Raimondo, J.C., Lemoine, J.M., Balmino, G., Biancale, R., Moynot, B., Marty, J.C., Barlier, F., Boudon, Y., 1993. Improvement of GRIM4 Earth gravity models using Geosat altimeter and SPOT-2 and ERS-1 tracking data. In: H. Montag, C. Reigber, (Editors) Geodesy and Physics of the Earth, Springer, Berlin, 75-78.

Tapley, B.D., Ries, J.C., Bettadpur, S., Chambers, D., Cheng, M., Condi, F., and Poole, S., 2007. The GGM03 Mean Earth Gravity Model from GRACE, EOS Transactions, American Geophysical Union 88(52), Fall Meeting Supplement, Abstract G42A-03.

Tapley, B., Watkins, M., Ries, J., Davis, G., Eanes, R., Poole, S., Rim, H., Schutz, B., Shum, C., Nerem, R., Lerch, F., Marshall, J. A., Klosko, S. M., Pavlis, N., Williamson R., 1996. The Joint Gravity Model 3, Journal of Geophysical Research, 101, B12, 28029-28049.

Editorial handling: R.B. Trumbull 